\title{
O impacto da pandemia de COVID-19 na saúde mental dos profissionais de saúde em município da baixada maranhense
}

The impacto f COVID-19 on the physical and emotional health of health professionals in the municipality of baixada maranhense

El impacto de la pandemia COVID-19 em la salud mental de los prodesionales de la salud en um município de baixada maranhão

Josuel Carlos Oliveira ORCID: https://orcid.org/0000-0002-4964-5374 Universidade Federal do Maranhão, Brasil E-mail: josuelcarlosoliveira@gmail.com Sanny Pinheiro Oliveira ORCID: https://orcid.org/0000-0002-4197-4368 Universidade Federal do Maranhão, Brasil E-mail: sanny.pinheiro@discente.ufma.br Getúlio Rosa dos Santos Junior ORCID: https://orcid.org/0000-0002-8930-4988 Universidade Federal do Maranhão, Brasil E-mail: getuliorosa.ufma@gmail.com

Laís da Hora Lucena Silva

ORCID: https://orcid.org/0000-0003-4558-6052 Universidade Federal do Maranhão, Brasil E-mail: laishlucena@gmail.com

Maria Augusta Ribeiro Gaspar ORCID: https://orcid.org/0000-0001-5056-3301 Universidade Federal do Maranhão, Brasil

E-mail: mariaargaspar@gmail.com

Cíntia Wélia Moraes Costa

ORCID: https://orcid.org/0000-0001-7119-4939 Secretaria Municipal de Saúde e Saneamento Básico de Pinheiro, Brasil

E-mail: cintiawelia@hotmail.com

Ricardo Daniel Santos Rodrigues

ORCID: https://orcid.org/0000-0002-9783-4110 Universidade Federal do Maranhão, Brasil

E-mail: ricardo.daniel@discente.ufma.br

Larissa Dias Parga

ORCID: https://orcid.org/0000-0002-6590-2502 Universidade Federal do Maranhão, Brasil

E-mail: larissa.parga@hotmail.com

Clarice Borges Carvalho

ORCID: https://orcid.org/0000-0001-6457-7923 Universidade Federal do Maranhão, Brasil

E-mail: clariceborges120@gmail.com

Lidiane Andréia Assunção Barros

ORCID: https://orcid.org/0000-0002-1614-3845

Universidade Federal do Maranhão, Brasil E-mail: lidiane.barros@ufma.br

Sara Fiterman Lima

ORCID: https://orcid.org/0000-0003-0015-3413 Universidade Federal do Maranhão, Brasil E-mail: sara.fiterman@ufma.br

Ariane Cristina Ferreira Bernardes Neves

ORCID: https://orcid.org/0000-0002-5258-1172 Universidade Federal do Maranhão, Brasil E-mail: ariane.bernardes@ufma.br

\section{Resumo}

Objetivo: Analisar o impacto da pandemia de COVID-19 na saúde mental dos profissionais da saúde no município de Pinheiro. Metodologia: Estudo descritivo, quantitativo e transversal, realizado com os profissionais envolvidos na assistência direta aos pacientes acometidos pela COVID-19 no município referido. Utilizou-se três instrumentos para 
a coleta dos dados (um questionário sociodemográfico; o inventário de ansiedade de Back-Bai; e a escala de Estresse Percebido) compilados em um formulário virtual na plataforma Google Formes, enviados aos participantes por meio de aplicativos de mensagens. Resultados: A amostra foi composta por 73 profissionais, dos quais, 79,45\% são mulheres, 72,60\% são católicos, 78,08\% recebem até 5 salários mínimos, 49,31\% de profissionais da enfermagem. Sobre à vivência de eventos marcantes no último ano, a maioria de 50,05\% negou, entretanto, 47,95\% admitiram essa vivência. No inventário de Ansiedade de Beck-Bai observou-se que 45,21\% foram classificados como sintomatologia mínima, 23,29\% leve, 17,81\% moderado e 13,70\% grave. Conclusão: Frequentes exposições a riscos de contaminação, grandes tomadas de decisões, longas jornadas de trabalho, falta de equipamentos de proteção individual, perda de colegas próximos ou familiares, apresentam-se como agravantes consideráveis para a saúde mental dos profissionais de saúde, e pouco se sabe das consequências desse sofrimento psicológico a longo prazo.

Palavras-chave: COVID-19; Profissionais da saúde; Saúde mental.

\begin{abstract}
Objective: To analyze the impact of the COVID-19 pandemic on the mental health of health professionals in the municipality of Pinheiro. Methodology: Descriptive, quantitative and cross-sectional study, carried out with professionals involved in direct care to patients affected by COVID-19 in the municipality mentioned. Three instruments were used for data collection (a sociodemographic questionnaire; the Back-Bai anxiety inventory; and the Perceived Stress scale) compiled in a virtual form on the Google Formes platform, sent to participants through messaging applications. Results: The sample consisted of 73 professionals, of which $79.45 \%$ are women, $72.60 \%$ are Catholic, $78.08 \%$ receive up to 5 minimum wages, $49.31 \%$ are nursing professionals. About the experience of remarkable events in the last year, the majority of 50.05\% denied it, however, $47.95 \%$ admitted this experience. In the Beck-Bai Anxiety Inventory it was observed that $45.21 \%$ were classified as minimal symptomatology, $23.29 \%$ mild, $17.81 \%$ moderate and $13.70 \%$ severe. Conclusion: Frequent exposure to contamination risks, major decision-making, long working hours, lack of personal protective equipment, loss of close colleagues or family members, are presented as considerable aggravating factors for the mental health of health professionals, and little the long-term consequences of this psychological suffering are know.
\end{abstract}

Keywords: COVID-19; Health professionals; Mental health.

\title{
Resumen
}

Objetivo: Analizar el impacto de la pandemia COVID-19 en la salud mental de los profesionales de la salud de la ciudad de Pinheiro. Metodología: Estudio descriptivo, cuantitativo y transversal, realizado con profesionales involucrados en la atención directa a pacientes afectados por COVID-19 en el municipio mencionado. Para la recolección de datos se utilizaron tres instrumentos (un cuestionario sociodemográfico; el inventario de ansiedad Back-Bai; y la escala de Estrés Percibido) compilados de forma virtual en la plataforma Google Formes, enviados a los participantes a través de aplicaciones de mensajería. Resultados: La muestra estuvo conformada por 73 profesionales, de los cuales $79.45 \%$ son mujeres, $72.60 \%$ son católicos, $78.08 \%$ reciben hasta 5 salarios mínimos, $49.31 \%$ son profesionales de enfermería. Sobre la experiencia de hechos notables en el último año, la mayoría del 50,05\% lo negó, sin embargo, el 47,95\% admitió esta experiencia. En el Inventario de Ansiedad de Beck-Bai se observó que el 45,21\% se clasificaron como sintomatología mínima, el 23,29\% leve, el 17,81\% moderada y el 13,70\% grave. Conclusión: La exposición frecuente a riesgos de contaminación, la toma de decisiones importantes, las largas jornadas laborales, la falta de equipo de protección personal, la pérdida de compañeros cercanos o familiares, se presentan como agravantes considerables para la salud mental de los profesionales de la salud, y poco a largo plazo. Se conocen a largo plazo las consecuencias de este sufrimiento psicológico.

Palabras clave: COVID-19; Profesionales de la salud; Salud mental.

\section{Introdução}

O vírus Sars-Cov-2 foi identificado na China no final de 2019, caracterizando-se por alto potencial de contágio e aumento exponencial de sua incidência. No início de 2020, a transmissão generalizada foi reconhecida pela Organização Mundial da Saúde (OMS), sendo declarada como uma pandemia sendo ainda considerada a maior emergência de saúde pública das últimas décadas (World Health Organization, 2020). O vírus causa um quadro clínico que varia de infecções assintomáticas a quadros respiratórios graves e essa condição passou a ser denominada de COVID-19 (Schmidt, Dantas \& Marziale, 2011).

A rápida disseminação do vírus, as incertezas sobre o controle da doença, além da imprevisibilidade acerca do tempo de duração da pandemia têm se apresentado como fatores condicionantes a agravos na saúde mental dos profissionais de saúde (Schmidt, Crepald, Bolze, Silva, \& Demenech 2020). Estudos realizados na China, demonstraram elevada prevalência de sinais e sintomas de sofrimento psicológico entre os trabalhadores que atuavam na linha de frente de assistência aos portadores 
(Zhang et al. 2020). Diante dessa realidade torna-se perceptível a necessidade de medidas que visem a promoção e prevenção de agravos à saúde mental desses profissionais (Ornell, Halpern, Kessler, \& Narvaez 2020).

Em relação ao Brasil, a curva de contágio tem se mantido em patamar elevado desde o início do contágio. Nesse cenário, os profissionais do sistema de saúde estão vivenciando a falta de equipamentos de proteção individual (EPIs), o risco de maior exposição ao contágio do vírus, a angústia nas tomadas de decisões, a dor de perder colegas de trabalho e pacientes, além do risco de transmissão do vírus aos familiares (Ornell, Schuch, Sordi, \& Kessler, 2020). Além disso, o medo de colapso do sistema de saúde, aumenta o risco de sofrimento emocional entre esses profissionais seja em decorrência da sobrecarga de trabalho, seja pelo autocuidado prejudicado em virtude da falta de tempo e consequente exaustão, gerando impacto significativo na saúde física e emocional destes profissionais (Sampaio, Oliveira, \& Pires, 2020).

Ressalta-se que, além do medo concreto da morte, a pandemia do COVID-19 trouxe implicações para estes profissionais em outras esferas, como na organização familiar, mudanças nas rotinas de trabalho e isolamento, levando a sentimentos de desamparo e abandono (Ornell, Halpern, Kessler, \& Narvaez, 2020).

A imprevisibilidade do desfecho da COVID-19 em curto prazo é um agravo à saúde física e mental dos profissionais da saúde e da população. Considera-se que o apoio social, a comunicação e a utilização de ajuda psicossocial e psicológica sem estigmatizá-los podem ser implementadas como estratégias para a redução do estresse emocional às quais esses indivíduos estão submetidos (Li, Wang, Xue, Zhao, \& Zhu, 2020).

Tendo em vista a realidade a que os trabalhadores da área da saúde estão expostos no contexto da pandemia, o presente estudo buscou responder o seguinte questionamento: Os profissionais que estão na linha de frente da COVID-19 no município de Pinheiro apresentam sinais de sofrimento mental ou estresse generalizado?

Assim, o presente estudo buscou analisar o impacto da pandemia de COVID- 19 na saúde mental dos profissionais da saúde no Município de Pinheiro, Maranhão, Brasil.

\section{Metodologia}

Estudo descritivo, quantitativo e transversal promovido pelo Projeto de Educação pelo Trabalho em Saúde Interprofissionalidade - (PET Saúde), da Universidade Federal do Maranhão (UFMA) -Campus Pinheiro, Maranhão. O estudo quantitativo utiliza o processamento eletrônico, onde os dados são organizados em tabelas e permite o teste das hipóteses estatísticas. Por conseguinte a ordenação lógica do trabalho fica facilitada e pode-se partir facilmente para a redação do relatório (Gil, 2002). Para composição da amostra foram incluídos no estudo os profissionais da saúde envolvidos na assistência direta aos pacientes acometidos pela COVID-19 (SarsCov-2) no município de Pinheiro, no Estado do Maranhão, sendo excluídos os trabalhadores do grupo de risco afastados das funções assistenciais, aqueles em gozo de férias ou de licença, e os em exercício de cargos administrativos exclusivamente. A amostra foi obtida por conveniência, em virtude das medidas de distanciamento social recomendadas durante a pandemia. A coleta de dados foi realizada durante o mês de maio de 2020 por meio de três instrumentos:

- Um questionário socidemográfico, elaborado pelos pesquisadores do subgrupo tutorial Hanseníase do PET Saúde, contendo as variáveis idade, sexo, estado civil, grau de escolaridade, crença, atuação profissional, hábitos de vida (tabagismo, etilismo e uso de medicamentos controlados) e presença de evento marcante no último ano;

- O Inventário de Ansiedade de Beck- BAI inicialmente criada por Beck, Epstein, Brown, e Steer, (1988), adaptado e validado no Brasil por Cunha, (2001). Contém vinte e uma (21) perguntas objetivas com categorias de sintomas e atividades avaliados em uma escala do tipo Likert, conforme a sua última semana. A soma dos escores identifica o grau de depressão, que pode ser classificado, em: mínimo (de 0 a 11 pontos), leve (de 12 a 19 pontos), moderado (de 20 a 35 pontos) e grave (de 36 a 63 pontos); 
- A Escala de Estresse Percebido (Perceived Stress Scale- PSS), construída por Cohen, Karmack, e Mermelsteinm (1983), é o instrumento mais utilizado para avaliar a percepção do estresse, já validada em mais de 20 países (Remor, 2006). A PSS avalia a percepção do indivíduo sobre o quanto imprevisíveis e incontroláveis lhe parecem os eventos de vida experimentados no último mês, podendo ser utilizada na população geral com, no mínimo, nível de escolaridade equivalente ao ensino fundamental completo (Cohen, \& Williamson, 1988). Trata-se de um instrumento composto por 14 itens, com opções de resposta variando de zero (0), com conotação para nunca, até a pontuação quatro (4), para representar sempre. As questões com conotação positiva nos itens 4, 5, 6, 7, 9, 10 e 13 têm sua pontuação somada invertida, de forma que o valor de zero tornase quatro, o valor de um (1) equivale a três (3), o valor de dois (2) se mantêm dois e o valor de quatro (4) torna-se zero (0). As demais questões são negativas e devem ser somadas conforme seu valor original. $\mathrm{O}$ total da escala é a soma das pontuações das 14 questões e seus escores podem variar de zero a 56 (Luft, Sanches, Mazo, Andrade, 2007).

Os instrumentos supracitados foram compilados em um formulário virtual na plataforma Google Formes juntamente com o Termo de Consentimento Livre e Esclarecido (TCLE e o Consentimento eletrônico, em obediência às recomendações da Resolução CNS N466/2012. O link deste formulário foi enviado para todos os participantes por meio do aplicativo de mensagens on line com prazo de reenvio de 48 horas.

Para a organização dos dados foi realizada a codificação de todas as variáveis dos instrumentos de coleta e elaborado um dicionário de dados (codebook) com estas codificações, bem como duas planilhas adicionais para a dupla digitação dos dados, empregando-se o Software MS-EXCEL. A análise dos dados foi obtida a partir dos números absolutos e relativos, e apresentados em formato de tabelas e gráficos.

A presente pesquisa está incluída nos objetivos do projeto de pesquisa vinculado ao Programa de Educação pelo Trabalho - PET Saúde - Interprofissionalidade, submetido à apreciação do Comitê de Ética em Pesquisa do Hospital Universitário da UFMA (CEP-HUUFMA) e aprovado segundo Certificado de Apresentação de Apreciação - CAAE: 29652520.0.0000.5087.

\section{Resultados e Discussão}

Dentre os 80 questionários respondidos, 07 foram excluídos, 02 por recusa manifesta no TCLE, 03 por duplicações no preenchimento e 02 devido aos respondentes não possuírem vínculo com o município pesquisado. Desta forma, foram analisados os dados preenchidos por 73 profissionais.

No que tange aos resultados do Inventário de Ansiedade de Beck- BAI da tabela 1, 33 (45,21\%) dos participantes foram classificados como sintomatologia mínima, 17 (23.29\%) como leve, 13 (17.81\%) como quadro moderado e 10 (13.70\%) como grave. Observa-se também na tabela 1 que, cumulativamente, 40 (54,79\%) dos profissionais apresentaram sintomatologia compatível com ansiedade e $33(45,21 \%)$ obtiveram pontuação compatível com ansiedade mínima. Os resultados são semelhantes ao estudo de Sampaio, Oliveira, e Pires (2020), realizado em Petrolina (PE) com 200 participantes, cuja classificação prevaleceu ansiedade leve 46 (23\%), já as categorias moderada e grave apresentaram menor concentração quando comparadas ao presente estudo, $16(8 \%)$ e $6(3 \%)$, respectivamente. 
Tabela 1: Prevalência de ansiedade, segundo a escala de ansiedade BECK-BAI, nos profissionais de saúde Pinheirenses em 2020 .

\begin{tabular}{lll}
\hline Escala beck-bai & Frequência & Percentual \\
Mínimo & 33 & 45.21 \\
Leve & 17 & 23.29 \\
Moderado & 13 & 17.81 \\
Grave & 10 & 13.70 \\
& & \\
\hline Total & $\mathbf{7 3}$ & $\mathbf{9 0 8}$ \\
\hline
\end{tabular}

Fonte: Autores \& Pinheiro (2020).

Quanto ao perfil sócio demográfico dos profissionais apresentados no quadro 1, $58(79,45 \%)$ são mulheres, na faixa etária correspondente de 30 a 39 anos em 37 (50,68\%), com estado civil predominante de casados em 28 (38,35\%) dos participantes. Quanto à religião, $53(72,60 \%)$ são católicos. No que se refere a renda mensal, $57(78,08 \%)$ referiram até 5 salários mínimos. Em relação a categoria profissional, 36 (49,31\%) eram profissionais da Enfermagem e a maioria dos profissionais apresentavam a titulação acadêmica de especialista. Quanto aos hábitos de vida, destaca-se a variável não tabagista em $72(98,63 \%)$ e não etilista em 48 (65,75\%). No que concerne a vivência de eventos marcantes no último ano, 38 $(52,05 \%)$ dos participantes referiram não ter sofrido eventos dessa natureza, entretanto considera-se significativo que 35 $(47,95 \%)$ afirmaram ter sofrido algum evento marcante no último ano. 
Quadro 1: Perfil sociodemográfico de profissionais de saúde com ansiedade, segundo a escala Beck-Bai, da rede de saúde de Pinheiro, Maranhão, Brasil, 2020.

\begin{tabular}{|c|c|c|c|c|c|c|}
\hline \multirow[t]{2}{*}{ Variáveis } & \multicolumn{4}{|c|}{ Escala Beck Bai } & \multirow[t]{2}{*}{ Total } & \multirow[t]{2}{*}{ Valor de $\mathbf{P}$} \\
\hline & Mínimo & Leve & Moderado & Grave & & \\
\hline \multicolumn{7}{|l|}{ Sexo } \\
\hline Feminino & 26 & 11 & 12 & 9 & 58 & 0.2922 \\
\hline Masculino & 7 & 6 & 1 & 5 & 15 & \\
\hline \multicolumn{7}{|l|}{ Idade } \\
\hline 20 a 29 anos & 7 & 5 & 6 & 6 & 24 & \\
\hline 30 a 39 anos & 18 & 10 & 6 & 3 & 37 & \\
\hline 40 a 49 anos & 7 & 1 & 1 & 0 & 9 & \\
\hline 50 a 59 anos & 1 & 1 & 0 & 1 & 3 & \\
\hline \multicolumn{7}{|l|}{ Estado civil } \\
\hline Solteiro & 11 & 5 & 7 & 2 & 25 & 0.9098 \\
\hline União estável & 10 & 2 & 0 & 5 & 17 & \\
\hline Casado & 11 & 10 & 4 & 3 & 28 & \\
\hline Separado & 1 & 0 & 2 & 2 & 3 & \\
\hline \multicolumn{7}{|l|}{ Crença Religiosa } \\
\hline Católico & 19 & 10 & 8 & 9 & 53 & 0.1223 \\
\hline Evangélica & 11 & 4 & 4 & 1 & 11 & \\
\hline Espírita & 2 & 3 & 1 & 0 & 4 & \\
\hline Agnóstico & 1 & 0 & 0 & 0 & 1 & \\
\hline \multicolumn{7}{|l|}{ Renda } \\
\hline Até 5 salário mínimo & 26 & 11 & 11 & 9 & 57 & 0.4412 \\
\hline Mais de 5 salários mínimos & 7 & 6 & 2 & 1 & 16 & \\
\hline \multicolumn{7}{|l|}{ Atuação Profissional } \\
\hline Enfermagem & 11 & 11 & 8 & 6 & 36 & 0.3692 \\
\hline Terapia ocupacional & 2 & 0 & 2 & 0 & 4 & \\
\hline Psicologia & 3 & 0 & 0 & 0 & 3 & \\
\hline Medicina & 3 & 0 & 1 & 0 & 4 & \\
\hline Técnicos de Enfermagem & 5 & 0 & 1 & 2 & 8 & \\
\hline Nutrição & 0 & 2 & 0 & 0 & 2 & \\
\hline Odontologia & 1 & 1 & 0 & 0 & 2 & \\
\hline $\mathrm{ACS}$ & 4 & 1 & 0 & 0 & 5 & \\
\hline Fonoaudiologia & 0 & 0 & 1 & 0 & 1 & \\
\hline Fisioterapia & 3 & 0 & 0 & 1 & 4 & \\
\hline Recepcionista & 0 & 1 & 0 & 1 & 2 & \\
\hline \multicolumn{7}{|l|}{ Tabagista } \\
\hline Sim & 1 & 0 & 0 & 0 & 1 & \\
\hline Não & 32 & 17 & 13 & 10 & 72 & \\
\hline \multicolumn{7}{|l|}{ Uso de Bebidas alcóolica } \\
\hline Sim & 12 & 5 & 5 & 3 & 25 & 0.8233 \\
\hline Não & 21 & 12 & 8 & 7 & 50 & \\
\hline \multicolumn{7}{|c|}{ Uso de Medicamento Controlado } \\
\hline $\operatorname{Sim}$ & 8 & 9 & 3 & 3 & 23 & 0.8195 \\
\hline Não & 25 & 8 & 10 & 7 & 50 & \\
\hline \multicolumn{7}{|c|}{ Evento marcante do Último Ano } \\
\hline $\operatorname{Sim}$ & 14 & 8 & 7 & 6 & 35 & 0.2869 \\
\hline Não & 19 & 9 & 6 & 4 & 38 & \\
\hline
\end{tabular}

Fonte: Autores. Pinheiro (2020).

Esse aumento percentual pode ter decorrido da pré-existente sobrecarga de trabalho potencializada com o advento da 
crise de Sars-Cov-2, observada na rede pública de serviço de saúde do município Pinheirense. Ademais, problemáticas surgidas com a crise da pandemia da COVID-19, tais como: o alto risco de infecção, escassez de equipamentos para proteção individual, exposição mais frequente ao adoecimento, morte e frustração corroboram para piora do quadro.

A população feminina analisada sob a perspectiva do inventário de Ansiedade de Beck- BAI se mostrou mais vulnerável a quadros de ansiedade de intensidades moderada e grave, enquanto a população masculina a intensidades mínimas e moderadas, achado semelhante aos resultados encontrados por Schmidt, Crepaldi, Bolze, Silva e Demenech (2020). No entanto, a diferença de amostragem das populações, segundo sexo, caracteriza-se como fator limitante desse resultado. A prevalência de respostas femininas vai ao encontro do pontuado na literatura, quando se associa essa variável à atuação profissional, haja vista que profissionais da enfermagem são compostos em sua maioria por essa população, relacionado a questões históricas e culturais do exercício laboral.

A média da variável renda mensal dos profissionais girou em torno de $\mathrm{R} \$ 4.415$ reais, sendo que, em 57 (78,08\%) da população detectada com ansiedade recebem até cinco salários mínimos. É sabido que essa variável, somada à carga horária laboral, tem influência na saúde mental e física dos profissionais. A carga horária de trabalho informada foi em torno de 46,6 horas semanais, isto é, com intervalo de 12 a 120 horas. Esses dados assemelham-se ao estudo realizado em onze hospitais da cidade de Londrina-PR, com amostragem de 211 trabalhadores, apontando 47,3 horas de carga horária semanal (D.P.=16; intervalo de 20 a 90 horas) e 66,4\% dos profissionais recebendo até três salários mínimos.

Quanto à variável idade, a amostra foi composta por jovens adultos, 61 (83,56\%) da amostra possui idade de 20 a 39 anos. Já quanto a variável tempo de atuação $(46,37 \%)$ dos profissionais possuem de 1 a 5 anos de serviço, seguidos dos profissionais com mais de 10 anos de atuação com concentração de (36,23\%). Os achados são corroborados pelos estudos de Dal'Bosco18 e Moura19, os quais afirmaram que os anos de trabalho podem ser considerados fator de risco para ansiedade, proporcionando um cotidiano intenso com os pacientes e equipe, um fator que se agrava ainda mais frente à pandemia do novo corona vírus.

As variáveis "tabagista", "uso de bebida alcoólica", "uso de medicamento controlado", e "evento marcante do último ano" que poderiam potencializar o quadro de ansiedade ou sinalizar consequências dessa alteração psicológica, apresentaram predomínio das suas respostas no questionário pontuadas como "não", em frequência correspondente à 72(98,63\%), 48 $(65,75 \%), 50(68,49 \%)$ e $38(52,05 \%)$, respectivamente. Quanto a evento marcante entre as poucas variáveis potencializa a hipótese de surgimento e/ou agravamento dos casos de ansiedade advindas da situação atípica trazida pela Covid-19. É válido pontuar, ainda, que as variáveis: estado civil, o uso de bebida alcoólica e uso de medicamento controlado não possuem significância com os resultados do inventário de ansiedade de BECK-BAI, visto que os valores ultrapassam o valor de $\mathrm{p}<$ 0,005, descritos no quadro 1 .

Para avaliar a ocorrência do estresse desencadeado pela pandemia de COVID-19 nos profissionais de saúde da linha de frente do município de Pinheiro/Maranhão, aplicou-se a Escala de Estresse Percebido (PSS). Segundo LEE (2012), a escala de estresse percebido é um instrumento que mensura o estresse psicológico do indivíduo e como os indivíduos avaliam os acontecimentos em suas vidas em relação ao estresse causado. Destaca-se que são adotados pontos de corte divididos em cinco categorias, assim, indivíduos que atinjam pontuação menor ou igual a 18 são considerados com estresse baixo, entre 19 e 24 estão categorizados com estresse normal, entre 25 e 29 apresentam estresse moderado, entre 30 e 35 considera-se estresse alto e acima de 35 refere-se a estresse muito alto. Os dados encontrados na pesquisa estão apresentados no Gráfico 1. 
Gráfco1: Escala De Estresse Percebido (PSS).

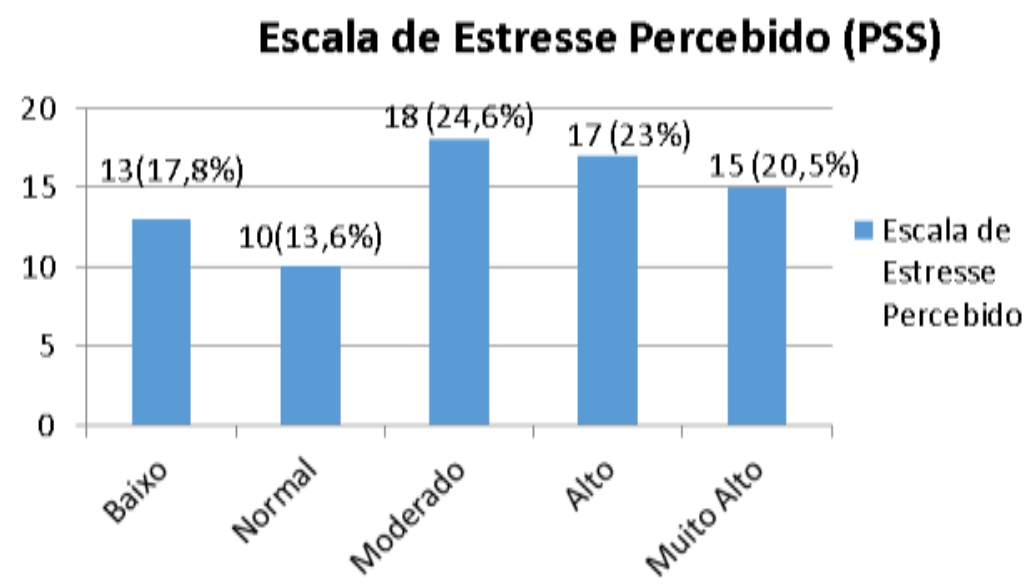

Fonte: Autores. Pinheiro (2020).

Em relação ao Estresse Percebido, 32 (43,83\%) profissionais foram classificados como alto e muito alto. Esse número fica ainda maior quando somado ao número de participantes que responderam sofrer moderado estresse, chegando a um total de 68,5\% (n=50). Tais resultados corroboram com o estudo de Dal'Bosco et al. (2020), realizado em Wuhan, na China, pelo qual comprovou-se níveis moderados a severos de estresse percebido pelo PSS em 59\% dos participantes. Em outro estudo produzido por Zhang, Wu, Zhao, e Zhang (2020), também se observou a confirmação de altas porcentagens - 73,4\% - de estresse percebido por profissionais de saúde que atuavam em hospitais de diferentes cidades chinesas, após a aplicação dessa mesma escala.

Destaca-se, ainda, que 58 (79,45\%) dos profissionais respondentes são do sexo feminino. Este achado se assemelha ao estudo de LAI et al. (2019), no qual profissionais mulheres, principalmente a categoria das enfermeiras, também apresentam mais suscetibilidade a manifestar estresse por decorrência do sono e também por estarem fortemente ligadas emocionalmente aos pacientes e seus desfechos conturbados provocados pela infecção do vírus. Ademais, ao avaliar o desempenho dos serviços de saúde na linha de frente do combate à pandemia do novo corona vírus, Xiao, Zhang, Kong, Li, e Yang (2020), aponta a associação do crescimento dos níveis de ansiedade e estresse à queda da auto eficácia, e identificaram que esses fatores induziam negativamente a qualidade do sono, principal causa de adoecimento físico e emocional desses profissionais. Para Huang, e Zhao (2020), o acúmulo de atividades fora do ambiente de atenção à saúde associado a uma pior qualidade de sono pode condicionar a ocorrência de casos graves, como o transtorno de estresse pós-traumático (TEPT).

Além disso, o estresse percebido e relatado por esses profissionais pode estar associado a múltiplos fatores, como a excessiva carga-horária de trabalho, aos procedimentos complexos realizados dentro do ambiente hospitalar, bem como a falta de EPIs. Tais aspectos, para KANG et al. (2020), foram percebidos como causa de um sofrimento psíquico, por parte dos profissionais. Associado a isso, a presença de estresse alto e muito alto encontrados por este estudo, e evidenciados no Gráfico 1, pode estar relacionada ao fato de que o aumento da intensidade do trabalho implica em menores períodos destinados ao descanso, o que estaria ligado a um estresse crônico e a um maior sofrimento psicológico, como observado por Li, Wang, Xue, Zhao, e Zhu (2020).

Outro fator observado por alguns estudos e que pode ter contribuído para o possível adoecimento mental dos profissionais de saúde foi o medo de transmitir o vírus à outras pessoas, as quais não faziam parte do serviço de saúde Kangl et al. (2020), e Marins et al (2020). Além disso, a COVID-19 apresentou inúmeros desafios aos trabalhadores da área de saúde possibilitando o desencadeamento do estresse que resulta em deixar esses profissionais ainda mais exaustos e propensos a 
cometer erros e contribuindo para uma assistência falha e fragmentada aos pacientes acometidos pela doença (Bao, Sun, Meng, Shi, \& Lu, 2019).

A pandemia da COVID-19 culminou no ápice de adoecimento mental dos profissionais de saúde por conta da rotina estressante, causada por um inimigo considerado invisível. Os desafios ocasionados pelo corona vírus estão distantes de serem cessados e o adoecimento dos profissionais de saúde da rede pública e privada podem trazer consequências a longo prazo.

Nesse sentido, a Organização Pan-Americana de Saúde [OPAS] (2020), bem como Prado, Peixoto, Silva, e Scalia (2020), recomendam que sejam disponibilizados serviços de auxílio psicológico neste período de crise, com a finalidade de acolher os profissionais de forma efetiva e intervir nas emoções de maneira empática, por meio da escuta ativa e qualificada. As intervenções devem ter foco no enfrentamento eficaz, na solução de problemas, na esperança e reflexões positivas com a intenção de provocar respostas psicoemocionais saudáveis. Associado a isso, Jiang et al. (2020), sugere uma ferramenta que permita prestar serviço de auxílio psicológico e, ao mesmo tempo, restringir o contato presencial, a qual seria realizada por meio das plataformas digitais, para o atendimento dos profissionais de saúde sob níveis de ansiedade e estresse elevados.

\section{Conclusão}

O presente estudo evidenciou que os profissionais que atuam na linha de frente do COVID-19 no município de Pinheiro, enfrentam uma série de fatores que provocam impactos na saúde mental. A população feminina analisada sob a perspectiva do inventário de Ansiedade se mostrou mais vulnerável a quadros de ansiedade variando entre moderada a grave, enquanto a população masculina apresentou-se com intensidades mínimas e moderadas. Contudo, a diferença de amostragem das populações, segundo sexo, caracterizou-se como um fator limitante. $\mathrm{O}$ resultado obtido sugere que esses profissionais enfrentam grande exposição ao risco de contaminação, difíceis tomadas de decisões, longas jornadas de trabalho, falta de equipamentos de proteção individual, além da complexa rotina que envolve o cuidado ao paciente crítico com Covid-19. Torna-se imprescindível que outras pesquisas sejam incentivadas, na investigação do impacto da pandemia na saúde física e mental dos profissionais que atuam na linha de frente, considerando uma amostragem ainda maior. Além da realização de intervenções psicológicas pelos serviços de saúde, a fim de minimizar os impactos negativos na saúde mental durante e póspandemia, momento este, em que as pessoas precisarão se adaptar e lidar com as perdas e transformações emocionais, sociais e econômicas, importantes.

\section{Agradecimentos}

Agradecemos ao Programa de Educação pelo Trabalho para a Saúde Pet-Saúde/Interprofissionalidade, edição 2018/2019 do Ministério da Saúde, por intermédio da Secretaria de Gestão do Trabalho e da Educação na Saúde (SGTES), pelo financiamento das bolsas para os integrantes do projeto que viabilizaram sua execução, e o apoio da Secretaria Municipal de Saúde de Pinheiro, e da Universidade Federal do Maranhão.

\section{Referências}

Bao, Y., Sun, Y., Meng, S., Shi, J., \& Lu, L. (2020). 2019-nCoV epidemic: address mental health care to empower society. The Lancet, 395(10224), e37-e38.

Barroso B. I. L., Souza, M. B. C. A; Bregalda, M.M., Lancman, S., \& Costa, V. B. B. (2020). A saúde do trabalhador em tempos de COVID-19: reflexões sobre a saúde, segurança e terapia ocupacional. Cad. Bras. Ter. Ocup. 28 (3). Recuperado em 20 maio, 2020 SciELO - Brasil - A saúde do trabalhador em tempos de COVID-19: reflexões sobre saúde, segurança e terapia ocupacional A saúde do trabalhador em tempos de COVID-19: reflexões sobre saúde, segurança e terapia ocupacional

Beck, A.T., Epstein, N., Brown, G., \& Steer, R. A. (1988). Um inventário para medir a ansiedade clínica: propriedades psicométricas. Revista de consultoria e psicologia clínica, 56(6), 893. 
Casemiro, F. G., Rodrigues, I. A., Dias, J. C., Alves, L. C. D. S., Inouye, K., \& Gratão, A. C. M. (2016). Impacto da estimulação cognitiva sobre depressão, ansiedade, cognição e capacidade funcional em adultos e idosos de uma universidade aberta da terceira idade. Revista Brasileira de Geriatria e Gerontologia, 19, 683-694.

Cohen, S., Kamarck, T., \& Mermelstein, R. (1983). A global measure of perceived stress. Journal of health and social behavior, 385-396.

Cohen, S., \& Williamson, G. (1988). Perceived stress in a probability sample of the United States In: Spacapan S, Oskamp S, editors. The social psychology of health: Claremont Symposium on applied social psychology.

Dal'Bosco, E. B., Floriano, L. S. M., Skupien, S. V., Arcaro, G., Martins, A. R., \& Anselmo, A. C. C. (2020). A saúde mental da enfermagem no enfrentamento da COVID-19 em um hospital universitário regional. Revista Brasileira de Enfermagem, 73.

Gil, A. C. (2002). Como elaborar projetos de pesquisa (Vol. 4, p. 175). São Paulo: Atlas.

Huang, Y., \& Zhao, N. (2020). Generalized anxiety disorder, depressive symptoms and sleep quality during COVID-19 outbreak in China: a web-based crosssectional survey. Psychiatry research, 288, 112954.

Jiang, X., Deng, L., Zhu, Y., Ji, H., Tao, L., Liu, L., \& Ji, W. (2020). Psychological crisis intervention during the outbreak period of new coronavirus pneumonia from experience in Shanghai. Psychiatry research, 286, 112903.

Kang, L., Li, Y., Hu, S., Chen, M., Yang, C., Yang, B. X., \& Liu, Z. (2020). A saúde mental dos médicos em Wuhan, China, lidando com o novo coronavírus de 2019. A Psiquiatria Lancet.

Lai, J., Ma, S., Wang, Y., Cai, Z., Hu, J., Wei, N., \& Hu, S. (2020). Factors associated with mental health outcomes among health care workers exposed to coronavirus disease 2019. JAMA network open, 3(3), e203976-e203976.

Lee, E. H. (2012). Review of the psychometric evidence of the perceived stress scale. Asian nursing research, 6(4), 121-127.

Li, S., Wang, Y., Xue, J., Zhao, N., \& Zhu, T. (2020). O impacto da declaração epidêmica do COVID-19 sobre as consequências psicológicas: um estudo sobre usuários ativos do Weibo. Revista internacional de pesquisa ambiental e saúde pública, 17(6), 2032.

Luft, C. D. B., Sanches, S. D. O., Mazo, G. Z., \& Andrade, A. (2007). Versão brasileira da Escala de Estresse Percebido: tradução e validação para idosos. Revista de Saúde Pública, 41, 606-615.

Marins, T. V. O, Crispim, C. G., da Silva Evangelista, D., do Carmo Neves, K., Fassarella, B. P. A., Ribeiro, W. A., \& da Silva, A. A. (2020). Enfermeiro na linha de frente ao COVID-19: A experiência da realidade vivenciada. Research, Society and Development, 9(8), e710986471-e710986471.

Moura, A., Lunardi, R., Volpato, R., Nascimento, V., Bassos, T., \& Lemes, A. (2018). Fatores associados à ansiedade entre profissionais da atenção básica. Revista Portuguesa de Enfermagem de Saúde Mental, (19), 17-26.

Organização Pan-Americana da Saúde. (2020). Folha informativa-COVID-19 (doença causada pelo novo coronavírus).

Ornell, F., Halpern, S. C., Kessler, F. H. P., \& Narvaez, J. C. M. (2020) The impactofthe COVID-19 pandemiconthe mental healthof healthcare professionals. Cadernos de Saúde Pública. Recuperado em 20 maio, 2020 https://doi.org/10.1590/0102-311X00063520.

Ornell, F., Schuch, J.B., Sordi, A. O., \& Kessler, F. H. P. (2020). "Medo pandemia" e COVID-19: carga e estratégias de saúde mental. Revista Brasileira de Psiquiatria, 42(3), 232-235.

Prado, A. D., Peixoto, B. C., Silva, A. M. B., \& Scalia, L. A. M. (2020). A saúde mental dos profissionais de saúde frente à pandemia do COVID-19: uma revisão integrativa. Revista Eletrônica Acervo Saúde, (46), e4128-e4128.

Remor, E. (2006). Psychometric properties of a European Spanish version of the Perceived Stress Scale (PSS). The Spanish journal of psychology, 9(1), 86-93.

Sampaio, L. R., de Oliveira, L. C., \& Pires, M. F. D. N. (2020). Empatia, depressão, ansiedade e estresse em Profissionais de Saúde Brasileiros. Ciencias Psicológicas, 14(2), 2215.

Schmidt B., Crepaldi, M. A., Bolze, S. D. A., Silva, L. N., \& Demenech, L. M. (2020). Saúde mental e intervenções psicológicas diante da pandemia do novo coronavírus (COVID-19). Estud. psicol. 37 (Campinas), Campinas. Recuperado em 18 maio, 2020 https://doi.org/10.1590/1982-0275202037e200063

Schmidt D. R. C., Dantas, R. A. S., \& Marziale, M. H. P. (2020). Ansiedade e depressão entre profissionais de enfermagem que atuam em blocos cirúrgicos. Rev. esc. enferm. USP, 45, 487-493, https://doi.org/10.1590/S0080-62342011000200026

World Health Organization. (2020). Coronavirus disease 2019 (COVID-19) Situation report - 63. https https://www.who.int/docs/defaultsource/coronaviruse/situation-reports/20200323-sitrep-63-covid-19.pdf?sfvrsn=d97cb6dd_2

Xiao, H., Zhang, Y., Kong, D., Li, S., \& Yang, N. (2020). The effects of social support on sleep quality of medical staff treating patients with coronavirus disease 2019 (COVID-19) in January and February 2020 in China. Medical science monitor: international medical journal of experimental and clinical research, 26, e923549-1.

Zhang, C., Yang, L., Liu, S., Ma, S., Wang, Y., Cai, Z., \& Zhang, B. (2020). Survey of insomnia and related social psychological factors among medical staff involved in the 2019 novel coronavirus disease outbreak. Frontiers in psychiatry, 11, 306.

Zhang, J., Wu, W., Zhao, X., \& Zhang, W. (2020). Recommended psychological crisis intervention response to the 2019 novel coronavirus pneumonia outbreak in China: a model of West China Hospital. Precision Clinical Medicine, 3(1), 3-8. 